\title{
TRANSPLANTE DE INTESTINO DELGADO
}

\author{
Small bowel transplantation \\ Rafael Antonio Arruda PÉCORA, André Ibrahim DAVID, André Dong LEE, Flávio Henrique GALVÃO, \\ Ruy Jorge CRUZ-JUNIOR, Luiz Augusto Carneiro D’ALBUQUERQUE
}

Trabalho realizado na Disciplina de Transplante de Órgãos do Aparelho Digestivo, Departamento de Gastroenterologia, Hospital das Clínicas da Faculdade de Medicina da Universidade de São Paulo, São Paulo, SP, Brasil.

DESCRITORES - Intestino delgado. Transplante.
RESUMO - Introdução: O transplante de intestino delgado, em razão de sua complexidade, apresentou evolução mais lenta que os demais órgãos sólidos. Diversos avanços permitiram sua aplicação clínica. Objetivo: Revisão da evolução do transplante de intestino delgado e seu estado atual. Método: levantamento bibliográfico nas bases de dados MEDLINE e ScIELO. Os termos usados como descritores foram: intestinal failure, intestinal transplant, small bowel transplant, multivisceral transplant. Foram analisados dados sobre evolução histórica, centros, indicações, tipos de enxertos, seleção e captação de órgãos, manejo pós-operatório, complicações e resultados. Conclusão: Apesar de desenvolvimento mais lento, o transplante intestinal é hoje a terapia para pacientes portadores de falência intestinal irreversível que apresentam complicações da nutrição parenteral. Envolve algumas modalidades: intestino delgado isolado, fígado-intestino, multivisceral e multivisceral modificado. Atualmente a sobrevida é semelhante aos demais órgãos sólidos. A maioria dos pacientes fica livre da nutrição parenteral.

\section{Correspondência:}

Rafael Antonio Arruda Pécora

E-mail: raapecora@yahoo.com.br

Fonte de financiamento: não há

Conflito de interesses: não há

Recebido para publicação: 24/10/2012

Aceito para publicação: 27/02/2013

HEADINGS - Small bowel. Transplantation.
ABSTRACT- Background: Small bowel transplantation evolution, because of its complexity, was slower than other solid organs. Several advances have enabled its clinical application. Aim: To review intestinal transplantation evolution and its current status. Method: Search in MEDLINE and ScIELO literature. The terms used as descriptors were: intestinal failure, intestinal transplantation, small bowel transplantation, multivisceral transplantation. Were analyzed data on historical evolution, centers experience, indications, types of grafts, selection and organ procurement, postoperative management, complications and results. Conclusion: Despite a slower evolution, intestinal transplantation is currently the standard therapy for patients with intestinal failure and life-threatening parenteral nutrition complications. It involves some modalities: small bowel transplantation, liverintestinal transplantation, multivisceral transplantation and modified multivisceral transplantation. Currently, survival rate is similar to other solid organs. Most of the patients become free of parenteral nutrition.

\section{INTRODUÇÃO}

$\mathrm{O}$ transplante de intestino delgado (TID) apresentou desenvolvimento mais lento em relação aos demais órgãos sólidos. Atualmente é a única possibilidade de cura para pacientes com falência intestinal que desenvolvem complicações relacionadas ao uso da nutrição parenteral. São procedimentos complexos em pacientes com condição clínica comprometida. Ele compreende uma série de modalidades cirúrgicas onde o órgão central a ser transplantado é o intestino delgado. Apesar de existir variação quando à terminologia, as modalidades frequentemente descritas são: intestino delgado isolado, multivisceral, multivisceral modificado e fígado-intestino. Existe a necessidade do emprego de imunossupressão intensa em razão da grande resposta imunológica ao enxerto. Dessa forma, infecções oportunistas e doenças proliferativas são mais prevalentes em relação aos demais transplantes de órgãos sólidos. Em razão da grande quantidade de tecido transplantada 
a doença enxerto versus hospedeiro também é mais prevalente. Soma-se o alto custo financeiro.

Este artigo tem por objetivo avaliar a evolução do TID e o seu estado atual.

\section{MÉTODO}

Foi realizado levantamento bibliográfico nas bases de dados MEDLNE e SciELO. Os termos usados como descritores foram: intestinal failure, intestinal transplant, small bowel transplant, multivisceral transplant. Foram analisados dados sobre evolução histórica, centros, indicações, tipos de enxertos, seleção e captação de órgãos, manejo pós-operatório, complicações e resultados.

\section{Histórico}

No início do século 20, Alexis Carrel realizou experimentos envolvendo transplantes de órgãos, incluindo o intestino delgado. Estudos pioneiros dirigidos a ele foram realizados em cães por Lillehei e cols. ${ }^{31} \mathrm{em}$ 1959. Os primeiros transplantes clínicos foram realizados em pacientes pediátricos em 1964 em Boston 5 . A tentativa com melhor documentação da época foi realizada por Lillehei et al. ${ }^{32} \mathrm{em}$ 1967. Os resultados iniciais precários foram em virtude de complicações técnicas, sepse e falta de esquemas imunossupressores eficazes para controlar a rejeição. Frente aos resultados desanimadores e com a introdução da nutrição parenteral por Dudrick et al. ${ }^{13} \mathrm{em}$ 1968, ocorreu perda no entusiasmo. Em 1968, Okumura ${ }^{38}$ realizou a primeira tentativa clínica na América Latina no Hospital das Clínicas da Faculdade de Medicina da Universidade de São Paulo.

O desenvolvimento da anestesiologia, da terapia intensiva e a introdução da ciclosporina ${ }^{9}$ em 1979, permitiram a melhora dos resultados dos transplantes de órgãos sólidos em geral. Em 1987, Thomaz Starzl et al. ${ }^{43}$ realizaram o primeiro transplante multivisceral com sobrevida, usando técnica descrita em 1960. Em 1989, Goulet et al. ${ }^{16}$ realizaram o primeiro transplante isolado de intestino delgado com sobrevida a longo prazo. Ao mesmo tempo, Grant et al. ${ }^{19}$ relataram o primeiro transplante combinado de intestino e fígado. Apesar do sucesso do uso da ciclosporina em outros tipos de transplante na década de 80 , os resultados no TID permaneciam com sucesso limitado.

A aplicação do tacrolimus nos anos 90 levou-o para outra esfera. Proporcionou melhor controle da rejeição, com melhora significativa nas sobrevidas de pacientes e enxertos. Em 2001 foi aceito nos EUA como modalidade terapêutica para pacientes com falência intestinal que apresentam complicações da nutrição parenteral. Nos últimos anos a sobrevida dos pacientes no primeiro ano se aproxima de outros órgãos sólidos.

\section{Centros}

Em razão da complexidade do procedimento, poucos centros realizaram TID no mundo.
Habitualmente os programas se desenvolveram em instituições onde os programas de transplante de fígado eram bem estabelecidos e com grande número de casos. Entre 2005 e 2007, apenas 28 centros no mundo relataram transplantes intestinais ao Intestinal Transplant Registry, realizando 389 transplantes intestinais em 377 pacientes $^{18}$. Apesar do pioneirismo inicial, o Brasil permaneceu por muitos anos sem um programa bem estabelecido. Observou-se alguns relatos recentes, porém sem sucesso ${ }^{11}$.

\section{Indicações}

A falência intestinal é condição clínica caracterizada pela redução da capacidade funcional do trato gastrointestinal para manter a digestão, absorção dos nutrientes e fluidos, necessários para a manutenção nos adultos, e ou para o crescimento e desenvolvimento nas crianças ${ }^{17}$. É resultado de grandes ressecções, doenças motoras e dos enterócitos, podendo existir associação dessas manifestações. Muitas das doenças não causam intrinsicamente perda de função, mas demandam múltiplas ressecções intestinais na sua história natural. Cerca de $60 \%$ dos casos ocorrem na população pediátrica. As causas mais frequentes são a enterocolite necrotizante, gastrosquise, atresia intestinal, volvo, pseudoobstrução e aganglionose. Entre os adultos, a isquemia mesentérica, doenças inflamatórias, enterite actínica, traumas e tumores são as causas mais comuns ${ }^{34}$.

Estima-se que 2-3 pessoas por milhão de habitantes por ano apresentarão falência intestinal ${ }^{23}$; destas, $15 \%$ serão candidatos ao TID ${ }^{24}$, por irreversibilidade da falência intestinal e complicações da nutrição parenteral. A mortalidade desse grupo é alta, atingindo $40 \%$ em cinco anos nos pacientes com menos de $50 \mathrm{~cm}$ de intestino delgado ${ }^{10}$, sendo decorrente de infecções dos catéteres, tromboses dos acessos e doença hepática .

Atualmente a falha da terapia nutricional parenteral, ou seja, pacientes que apresentam complicações são candidatos ao TID. As complicações mais aceitas como indicações são ${ }^{48}$ : trombose de dois dos seis acessos venosos principais; doença hepática; episódios de infecções relacionadas ao cateter (dois ou mais ao ano, fungemia, choque ou insuficiência respiratória); alterações do crescimento e desenvolvimento em crianças, e alterações hidroeletrolíticas refratárias.

No transplante multivisceral, existem outras indicações ${ }^{48}$ : catástrofes abdominais, tumores benignos ou malignos de baixo grau do eixo mesentérico e trombose difusa do sistema mesentericoportal.

As catástrofes abdominais compreendem situações crônicas debilitantes causadas por trauma abdominal, pancreatite aguda grave, ressecções intestinais extensas ou múltiplas intervenções abdominais, que levam à síndrome do intestino curto, múltiplas fístulas enterocutâneas, obstruções intestinais crônicas ou trombose vascular mesentérica difusa. A substituição 
completa de todas as vísceras da cavidade abdominal (transplante multivisceral) pode ser a única alternativa de reestabelecimento da normalidade fisiológica ${ }^{37}$.

Tromboses complexas do sistema venoso mesentéricoportal também podem ser indicações do transplante multivisceral. A situação mais comumente envolvida nesse contexto é o transplante de fígado com trombose portal. No passado, a presença de trombose portal nos candidatos ao transplante de fígado já foi contraindicação ao procedimento. Apesar de não limitá-lo, adiciona dificuldades técnicas importantes e morbimortalidade maior. A classificação proposta por Yerdel ${ }^{50}$, pode orientar a decisão cirúrgica: grau I - comprometimento de menos de $50 \%$ da luz, com pequena extensão para a veia mesentérica superior; grau II - comprometimento de mais de $50 \%$ da luz, incluindo obstrução total, porém com pequena extensão para a veia mesentérica superior; grau III oclusão completa da veia porta e da veia mesentérica superior proximal; grau IV - oclusão completa da veia porta e da veia mesentérica superior distal.

As alternativas para revascularização portal do enxerto hepático podem ser a simples remoção do trombo, passando por enxertos para a veia mesentérica superior e varizes. Nas tromboses grau IV, a arterialização portal, enxerto para a veia renal e hemitransposição cavoportal ${ }^{39}$ são alternativas. Permitem a vascularização do órgão, sem descomprimir o território portal. A manutenção da hipertensão portal (ascite, hemorragia digestiva), além do desenvolvimento de trombose da veia cava inferior e insuficiência renal, são responsáveis por mortalidade hospitalar elevada ${ }^{39}$ (33\%) e sobrevida a longo prazo ruim (aproximadamente 35\% em cinco anos) $)^{42}$. O transplante multivisceral tem sido proposto como alternativa para tromboses complexas do sistema mesentérico portal, mesmo na ausência de falência hepática ou intestinal ${ }^{47}$.

Uma variedade de tumores pode envolver o eixo mesentérico portal e celíaco. Tumores neuroendócrinos e adenocarcinoma do pâncreas, e tumores desmóides são alguns dos exemplos. A ressecção por vezes é arriscada, em outras impossível sem comprometimento da vascularização das vísceras abdominais. O transplante de intestino isolado, incluindo o autotransplante e multivisceral têm sido propostos como alternativas para essas situações ${ }^{36}$. Em razão da recidiva precoce e grave no adenocarcinoma, o transplante para os tumores desmóides e neuroendócrinos bem diferenciados tem sido mais aceito ${ }^{36}$. No entanto as casuísticas são pequenas e aprimoramento dos critérios de seleção é necessário.

Pacientes dependentes de nutrição parenteral sem complicações não são candidatos ao transplante intestinal. Existem relatos de pacientes mantidos em nutrição parenteral por muitos anos. No entanto, a qualidade de vida deles é questionada, além do alto custo para manutenção da nutrição. Estudos avaliando a qualidade de vida ${ }^{44}$, antes e depois do transplante, com questionários validados, mostraram melhora em diversos aspectos, incluindo ansiedade, depressão e autoimagem. Não existem estudos controlados comparando a nutrição parenteral com o TID.

\section{Seleção do tipo de enxerto}

O TID envolve algumas modalidades onde 0 órgão central a ser transplantado é o intestino delgado. A seleção dos órgãos a serem incluídos vai depender da doença de base, qualidade dos demais órgãos abdominais, presença e gravidade de doença hepática e número de operações abdominais pregressas.

$O$ enxerto de intestino delgado isolado (Figura 1) é indicado na presença de falência intestinal irreversível na ausência de disfunção hepática severa. A determinação da gravidade da doença hepática e irreversibilidade é realizada de forma mais segura através da biópsia hepática. A presença de fibrose em ponte ou cirrose indica a necessidade de substituição do fígado. Estudo mostrou associação entre os níveis de bilirrubinas, contagem de plaquetas e nível de albumina com a presença de falência hepática em crianças $^{28} \mathrm{em}$ nutrição parenteral. A irrigação arterial é estabelecida através de anastomose da artéria mesentérica superior do enxerto com a aorta. A drenagem venosa pela veia mesentérica superior para a veia cava inferior ou para o sistema mesentérico portal. Estudo ${ }^{6}$ mostrou não haver diferença de sobrevida, no entanto, a incidência cumulativa dos episódios de infecção por germes do trato gastrointestinal foi maior no grupo com drenagem sistêmica, sugerindo papel protetor hepático. Em todas as modalidades é realizada uma ileostomia para vigilância endoscópica, facilitando o diagnóstico de rejeição e de distúrbios perfusionais.

$\mathrm{Na}$ presença de doença hepática irreversível, o fígado deverá ser incluído no enxerto. Esse grupo de pacientes passa a competir pelos enxertos hepáticos já escassos. O sistema MELD/PELD utilizado para alocação de fígados em muitos países, no entanto, não é adequado para esses pacientes, que apresentam risco de óbito em lista 3,6 vezes maior que pacientes com mesmo MELD/PELD que aguardam apenas o fígado ${ }^{22}$. Dados dos EUA mostram que $74 \%$ dos pacientes candidatos ao transplante intestinal necessitam de um fígado associado ${ }^{15}$. Aperfeiçoamento dos modelos de alocação e o encaminhamento precoce para o TID pode ser uma solução para esse problema. Os enxertos podem ser implantados de forma separada, ou de modo mais conveniente, em bloco. Para manutenção do fígado e intestino em bloco, é necessária a inclusão do arco pancreatoduodenal no enxerto (Figura 2). Evitamse assim as dissecções biliar e portal, que podem ser difíceis em crianças pequenas. A irrigação arterial é estabelecida através de um conduto arterial com a aorta. A drenagem venosa através das veias hepáticas para a veia cava inferior, ao modo do transplante hepático. Para drenagem venosa das vísceras remanescente é necessária a criação de um shunt para a veia cava ou sistema portal. 
Na falência de múltiplas vísceras abdominais o enxerto a ser empregado é o multivisceral (Figura 3). É necessária a evisceração completa da cavidade abdominal. São transplantados em bloco: estômago, duodeno, pâncreas, intestino delgado e fígado. A irrigação arterial é estabelecida através das artérias mesentérica superior e tronco celíaco do enxerto através de um conduto arterial com a aorta e drenagem venosa através das veias hepáticas para a veia cava inferior. O tubo digestivo é restabelecido através de anastomose do esôfago com o estômago do enxerto.

No transplante multivisceral modificado (Figura 4), o fígado do receptor é mantido. São transplantados em bloco: estômago, duodeno, pâncreas e intestino delgado. Doenças motoras do aparelho digestivo como pseudoobstrução intestinal ou doenças inflamatórias como Crohn podem ser indicações. A irrigação arterial, e continuidade do tubo digestivo são estabelecidos como no transplante multivisceral. A drenagem venosa é feita através da veia porta.

Controvérsias existem em relação à inclusão do cólon e baço nos enxertos. Estudo ${ }^{26}$ mostrou que a inclusão de segmentos colônicos não acrescentou qualquer morbidade, mas trouxe benefícios de continência apenas na faixa pediátrica. Em relação ao baço, em estudo do mesmo grupo ${ }^{27}$, houve tendência de benefício imunológico, sem alterar a incidência de doença enxerto versus hospedeiro.

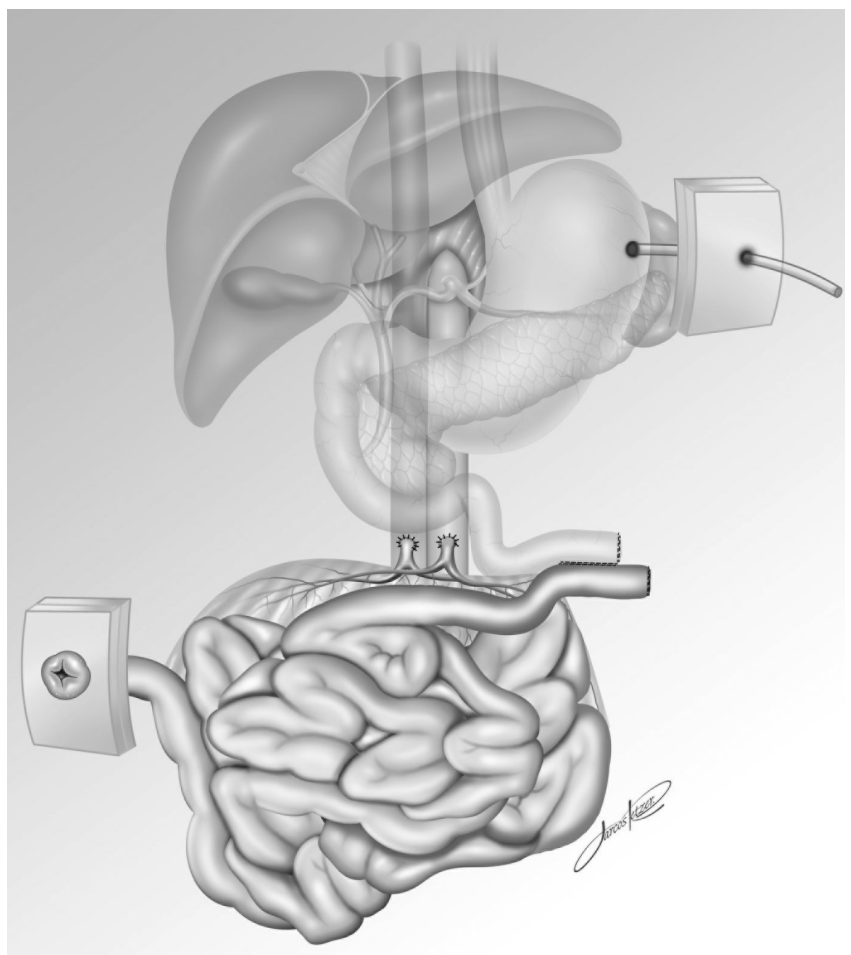

FIGURA 1 - Intestino delgado isolado

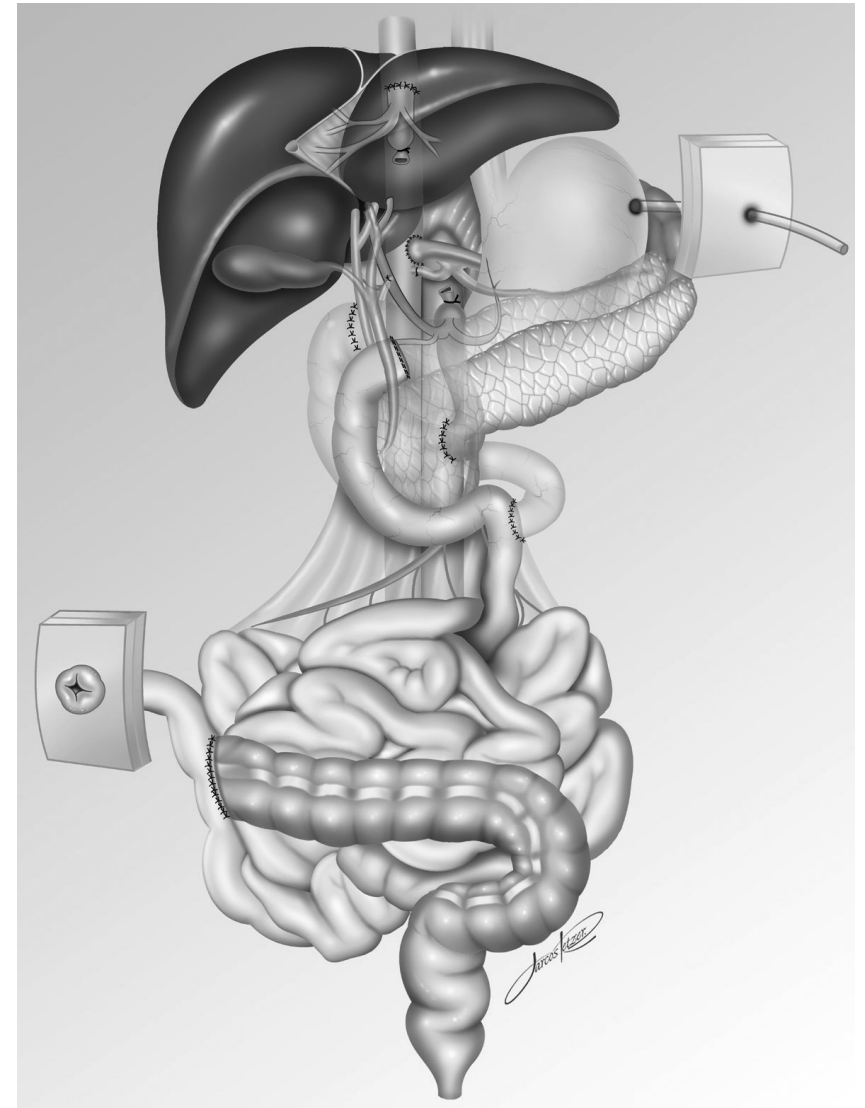

FIGURA 2 - Fígado-intestino

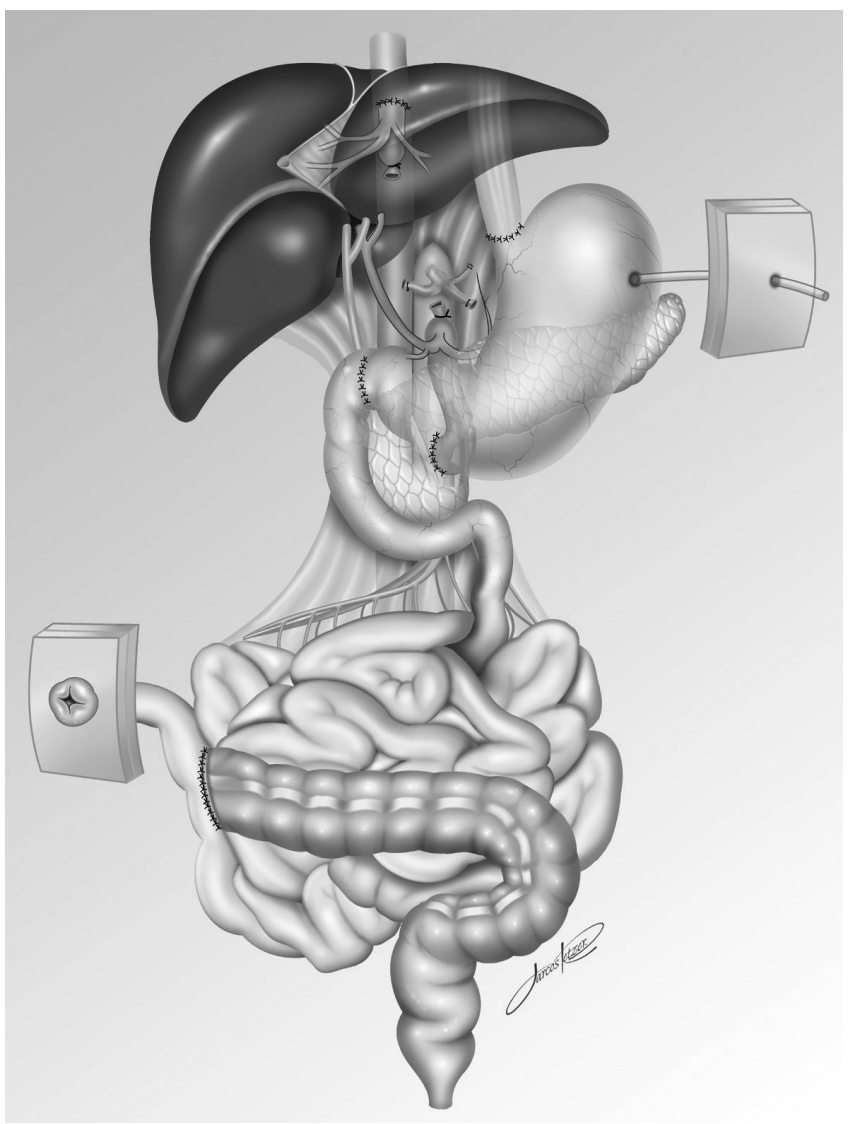

Figura 3 - Multivisceral 


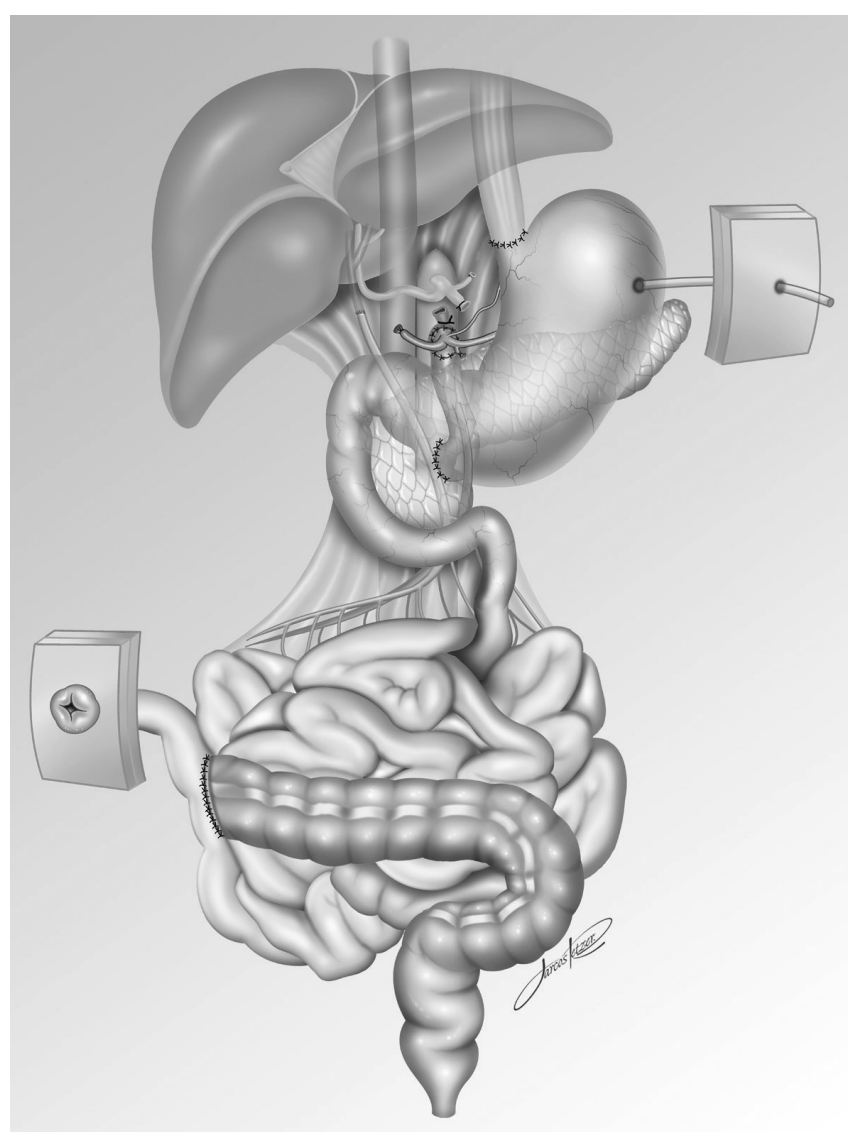

FIGURA 4 - Multivisceral modificado

Doadores, captação de enxertos e preservação

A seleção dos enxertos provenientes de doadores falecidos segue critérios semelhantes ao fígado ${ }^{48} \mathrm{com}$ algumas alterações. São utilizados preferencialmente mais jovens e com pouca ou nenhuma droga vasoativa. Os pacientes portadores de intestino curto apresentam a cavidade abdominal retraída, sendo necessária a utilização de doadores menores (30 a 40\%). Dá-se preferência à identidade $A B O$. Com o desenvolvimento de drogas eficazes para profilaxia e tratamento do citomegalovírus, doadores com sorologia positiva são aceitos, evitando-seapenas para receptores comsorologia negativa. Descontaminação do trato gastrointestinal e a utilização de anticorpos anti-linfócitos nos doadores não mostraram benefícios relacionados à infecção, episódios de rejeição ou incidência de doença enxerto versus hospedeiro. Habitualmente serão doadores também de fígado e pâncreas. Frente à circulação sanguínea compartilhada, a captação simultânea desses enxertos pode ser um desafio. Estudo ${ }^{1}$ demonstrou a possibilidade de execução do procedimento sem comprometimento dos enxertos.

A solução da Universidade de Wisconsin tem sido considerada o padrão-ouro para preservação de órgãos do aparelho digestivo, não sendo diferente para o intestino. No entanto, existem relatos do uso das soluções Celsior ${ }^{30}$ e HTK $^{33}$ no TID com resultados comparáveis à solução da Universidade de Wisconsin para períodos de isquemia de até $8 \mathrm{~h}$.

\section{Manejo pós-operatório e complicações}

No pós-operatório, além das complicações cirúrgicas (hemorragias, fístulas, deiscências e infecções de parede), podem ocorrer episódios de rejeição, infecções oportunistas e reabilitação nutricional.

O maior obstáculo ao transplante intestinal é a rejeição do enxerto. É o principal fator de morbimortalidade no transplante intestinal. Apresentando impacto negativo na sobrevida do enxerto ${ }^{18}$. A rejeição celular aguda ocorre em 50 a $75 \%$ dos pacientes, sendo mais comum nos primeiros 90 dias $^{3}$. A rejeição crônica ocorre em $15 \%$ dos pacientes ${ }^{3}$.

Diversos esquemas de imunossupressão e estratégias (irradiação do enxerto, infusão de células da medula do doador) foram utilizados sem impacto ${ }^{3}$. Melhores resultados foram obtidos com a terapia de indução com anticorpos anti-linfócitos, monoclonais ou policlonais, sendo utilizada na maioria dos centros $3,14,29,46$. As drogas mais utilizadas para indução são timoglobulina, alemtuzumab, basiliximab e daclizumab. A imunossupressão de manutenção é realizada com tacrolimus, mantendo-se no primeiro mês níveis de 12 a $15 \mathrm{ng} / \mathrm{ml}$ e redução para 8-12 $\mathrm{ng} / \mathrm{ml}$ após esse período inicial ${ }^{48}$. Como nos demais transplantes de órgãos abdominais, corticosteróides também são utilizados, sendo removidos de acordo com o tipo de enxerto e preferência de cada centro. A prova cruzada pode ajudar na individualização da imunossupressão. Estudo com 130 transplantes intestinais, a prova cruzada foi positiva em $18 \%$, estando associada ao aumento da frequência e gravidade dos episódios de rejeição ${ }^{7}$, sendo mais importante nos enxertos de intestino isolado.

O diagnóstico da rejeição celular aguda é realizado através do quadro clínico, achados endoscópicos e anatomia patológica. $\mathrm{Na}$ vigência de rejeição celular aguda os pacientes são bastante sintomáticos, apresentando febre, dor abdominal, vômitos, aumento de volume pela estomia e sangramento gastrointestinal. O comprometimento inicia-se pelo íleo terminal. A ileostomia de rotina facilita a avaliação endoscópica e realização de biópsias. A vigilância endoscópica, com magnificação de 100x, é realizada duas a três vezes por semana nos três primeiros meses, sendo realizada uma vez por mês a partir de então e conforme a necessidade ${ }^{48}$. O fechamento da ileostomia varia conforme o centro e o tipo de transplante (enxertos com ou sem fígado), sendo realizada entre três a 12 meses $^{48}$. Uma série de achados endoscópicos pode estar associada à rejeição aguda: eritema da mucosa, congestão, encurtamento e achatamento dos vilos, friabilidade e ulcerações ${ }^{25}$. A endoscopia isolada apresenta sensibilidade de apenas $52 \%$, porém especificidade de $93 \%{ }^{25}$. O padrão-ouro para o diagnóstico da rejeição celular aguda é a histologia. $\mathrm{Na}$ suspeita de rejeição diversas biópsias devem ser realizadas, pois a lesão pode poupar alguns segmentos. Estudo realizado com a avaliação de aproximadamente 3.000 biópsias $^{49}$, identificou quatro parâmetros principais 
relacionados à rejeição, que permitem análise quantitativa ou semi, e podem ser facilmente identificados por diferentes patologistas: distorção arquitetural, lesão epitelial da cripta intestinal, número de apoptoses porcripta e infiltrado linfocitário na lâmina própria. O diagnóstico diferencial deve ser feito com infecções oportunistas (citomegalovírus, adenovírus), doenças linfoproliferativas e outras enterites. Conforme a histologia, a rejeição pode ser classificada em indeterminada, leve, moderada ou grave. Marcadores não invasivos (citrulina, calprotectina) para o diagnóstico de rejeição ainda não fazem parte da rotina clínica, em razão da baixas especificidades ${ }^{8,12}$. Episódios de rejeição leve podem ser tratados com pulsos de esteróides. Episódios moderados ou graves necessitam de terapia com anticorpos anti-linfocitos (alemtuzumab, timoglobulina). A rejeição crônica é manifestação grave, sem tratamento, que comumente leva à perda do enxerto. Com mecanismos pouco esclarecidos, caracteriza-se pela fibrose submucosa e arteriopatia obliterativa. Está associada a episódios de rejeição aguda no primeiro mês, gravidade e número dos episódios de rejeição aguda e enxertos que contenham apenas intestino delgado ${ }^{40}$.

O objetivo principal do transplante intestinal é o restabelecimento da nutrição pelo trato digestivo. Até $90 \%$ dos pacientes submetidos ao transplante intestinal podem ficar livres da nutrição parenteral ${ }^{2}$. No entanto, é necessária a realização de reabilitação intestinal, uma vez que os enxertos podem apresentar graus variáveis de insuficiência em razão de lesão de isquemiareperfusão, denervação e episódios de rejeição. Existem diversos esquemas para o processo de adaptação ${ }^{21}$, sem superioridade clara de qualquer. Habitualmente envolvem manutenção inicial em nutrição parenteral com transição gradual para enteral. Dietas enterais elementares, oligoméricas ou poliméricas são administradas quando o enxerto exibir sinais de funcionamento. Os lipídeos são introduzidos lentamente (após quatro semanas) pelo risco de desenvolvimento de ascite quilosa. Em razão da absorção portal, os triglicerídeos de cadeia média são utilizados como principal fonte.

As infecções são manifestações universais no TID. Infecções de catéteres, condição clínica ruim, imunossupressão intensa, procedimentos cirúrgicos de grande porte e translocação bacteriana intestinal após rejeição são responsáveis pelo quadro. São a principal causa de mortalidade direta. Relatam-se infecções bacterianas em $94 \%$, virais em $67 \%$ e fúngicas em $28 \%$ dos pacientes ${ }^{20}$. As virais são responsáveis por $60 \%$ das perdas dos enxertos ${ }^{41}$. Infecções por citomegalovírus ocorrem em até $40 \%$ dos transplantados, em média com três meses ${ }^{41}$. A infecção por vírus Epstein-Barr é associada ao desenvolvimento de doença linfoproliferativa em $12,5 \%$ dos transplantados ${ }^{4}$, ocorrendo em média com 5,5 meses, sendo mais comum na faixa pediátrica. Compromete o enxerto intestinal em 71\% dos $\operatorname{casos}^{4} \mathrm{e}$ afeta de forma importante a sobrevida. A monitoração regular do citomegalovírus e do Epstein-Barr através de $P C R$, e o tratamento e profilaxia agressivos com antivirais e imunoglobulinas são rotinas nos serviços de TID.

A doença enxerto versus hospedeiro é pouco comum nos transplantes renal, pancreático e hepático; no entanto, é esperada no transplante intestinal em razão da grande carga linfocitária que é transplantada. É mais comum no transplante multivisceral. Em estudo de $2004^{35}$, 5,6\% dos pacientes tiveram confirmação histológica, com mortalidade de aproximadamente $9 \%$.

\section{Resultados do transplante intestinal}

Entre abril de 1985 a maio de 2007, 1720 transplantes intestinais foram registrados ${ }^{18}$, sendo: 746 intestino isolado, 594 fígado-intestino e 380 multivisceral. A Universidade de Pittsburgh já atingiu mais de 500 transplantes ${ }^{3}$, com sobrevida atuarial de $85 \%$ em um ano e $61 \%$ em cinco anos. A sobrevida do enxerto foi de $80 \%$ em um ano e $50 \%$ em cinco anos. Séries de outros centros $^{45}$ mostram atualmente sobrevida de $78-85 \%$ em um ano e $56-61 \%$ entre cinco e dez anos.

\section{CONCLUSÕES}

O transplante intestinal é hoje terapia bem estabelecida para pacientes com insuficiência intestinal irreversível que apresentam complicações da nutrição parenteral, com sobrevidas de enxertos e pacientes se aproximando dos outros órgãos sólidos. A abordagem multidisciplinar dos pacientes com falência intestinal, o encaminhamento precoce para as listas de transplantes, o diagnóstico precoce e tratamento agressivo das infecções oportunistas virais, a utilização de terapia de indução de imunossupressão com anticorpos e o diagnóstico e tratamento precoce da rejeição aguda do enxerto foram importantes para os melhores resultados. A seleção adequada dos receptores e o cuidado rigoroso no pós-operatório são fundamentais para o sucesso deste transplante.

\section{REFERÊNCIAS}

1. Abu-Elmagd, K., J. Fung, et al. (2000). "Logistics and technique for procurement of intestinal, pancreatic, and hepatic grafts from the same donor." Ann Surg 232(5): 680-687.

2. Abu-Elmagd, K. M. (2006). "Intestinal transplantation for short bowel syndrome and gastrointestinal failure: current consensus, rewarding outcomes, and practical guidelines." Gastroenterology 130(2 Suppl 1): S132-137.

3. Abu-Elmagd, K. M., G. Costa, et al. (2009). "Five hundred intestinal and multivisceral transplantations at a single center: major advances with new challenges." Ann Surg 250(4): 567-581.

4. Abu-Elmagd, K. M., G. Mazariegos, et al. (2009). "Lymphoproliferative disorders and de novo malignancies in intestinal and multivisceral recipients: improved outcomes with new outlooks." Transplantation 88(7): 926-934.

5. Alican, F., J. D. Hardy, et al. (1971). "Intestinal transplantation: laboratory experience and report of a clinical case." Am J Surg 121(2): 150-159.

6. Berney, T., T. Kato, et al. (2002). "Portal versus systemic drainage of small bowel allografts: comparative assessment of survival, function, rejection, and bacterial translocation." J Am Coll Surg 195(6): 804-813. 
7. Bond, G., J. Reyes, et al. (2000). "The impact of positive T-cell lymphocytotoxic crossmatch on intestinal allograft rejection and survival." Transplant Proc 32(6): 1197-1198.

8. Cagnola, H., R. Scaravonati, et al. (2010). "Evaluation of calprotectin level in intestinal content as an early marker for graft rejection." Transplant Proc 42(1): 57-61.

9. Calne, R. Y., K. Rolles, et al. (1979). "Cyclosporin A initially as the only immunosuppressant in 34 recipients of cadaveric organs: 32 kidneys, 2 pancreases, and 2 livers." Lancet 2(8151): 1033-1036.

10. Chan, S., K. C. McCowen, et al. (1999). "Incidence, prognosis, and etiology of end-stage liver disease in patients receiving home total parenteral nutrition." Surgery 126(1): 28-34.

11. da Silva, R. F., A. C. de Paula, et al. (2008). "Report of initial experience in small bowel transplantation at Sao Jose do Rio Preto Medical School Hospital." Transplant Proc 40(3): 827-829.

12. David, A. I., L. A. Szutan, et al. (2008). "[Critical value of citrulline for complications of intestinal transplant graft]." Rev Assoc Med Bras 54(5): 426-429.

13. Dudrick, S. J., D. W. Wilmore, et al. (1968). "Long-term total parenteral nutrition with growth, development, and positive nitrogen balance." Surgery 64(1): 134-142.

14. Fryer, J. P. (2007). "Intestinal transplantation: current status." Gastroenterol Clin North Am 36(1): 145-159, vii.

15. Fryer, J. P. (2008). "The current status of intestinal transplantation." Curr Opin Organ Transplant 13(3): 266-272.

16. Goulet, O., Y. Revillon, et al. (1992). "Two and one-half-year followup after isolated cadaveric small bowel transplantation in an infant." Transplant Proc 24(3): 1224-1225.

17. Goulet, O. and F. Ruemmele (2006). "Causes and management of intestinal failure in children." Gastroenterology 130(2 Suppl 1): S16-28.

18.Grant, D. (2007). "Intestinal transplant registry." from www. intestinaltransplant.org.

19.Grant, D., W. Wall, et al. (1990). "Successful small-bowel/liver transplantation." Lancet 335(8683): 181-184.

20.Guaraldi, G., S. Cocchi, et al. (2005). "Outcome, incidence, and timing of infectious complications in small bowel and multivisceral organ transplantation patients." Transplantation 80(12): 17421748.

21.Gupte, G. L. and S. V. Beath (2009). "Update on intestinal rehabilitation after intestinal transplantation." Curr Opin Organ Transplant 14(3): 267-273.

22. Horslen, S. (2004). "Organ allocation for liver-intestine candidates." Liver Transpl 10(10 Suppl 2): S86-89.

23. Howard, L. and N. Hassan (1998). "Home parenteral nutrition. 25 years later." Gastroenterol Clin North Am 27(2): 481-512.

24. Howard, L. and M. Malone (1996). "Current status of home parenteral nutrition in the United States." Transplant Proc 28(5): 2691-2695.

25. Kato, T., J. J. Gaynor, et al. (2006). "Zoom endoscopic monitoring of small bowel allograft rejection." Surg Endosc 20(5): 773-782.

26. Kato, T., G. Selvaggi, et al. (2008). "Inclusion of donor colon and ileocecal valve in intestinal transplantation." Transplantation 86(2): 293-297.

27. Kato, T., A. G. Tzakis, et al. (2007). "Transplantation of the spleen: effect of splenic allograft in human multivisceral transplantation." Ann Surg 246(3): 436-444; discussion 445-436.

28. Kaufman, S. S., M. Pehlivanova, et al. (2010). "Predicting liver failure in parenteral nutrition-dependent short bowel syndrome of infancy." J Pediatr 156(4): 580-585 e581.

29. Langnas, A. N. (2004). "Advances in small-intestine transplantation." Transplantation 77(9 Suppl): S75-78.
30. Lauro, A., F. Di Benedetto, et al. (2005). "Multivisceral harvest with in vivo technique: methods and results." Transplant Proc 37(6): 2425-2427.

31. Lillehei, R. C., B. Goott, et al. (1959). "The physiological response of the small bowel of the dog to ischemia including prolonged in vitro preservation of the bowel with successful replacement and survival." Ann Surg 150: 543-560.

32. Lillehei, R. C., Y. Idezuki, et al. (1967). "Transplantation of stomach, intestine, and pancreas: experimental and clinical observations." Surgery 62(4): 721-741.

33. Mangus, R. S., A. J. Tector, et al. (2008). "Comparison of histidinetryptophan-ketoglutarate solution and University of Wisconsin solution in intestinal and multivisceral transplantation." Transplantation 86(2): 298-302.

34. Mazariegos, G. V. (2009). "Intestinal transplantation: current outcomes and opportunities." Curr Opin Organ Transplant 14(5): 515-521.

35. Mazariegos, G. V., K. Abu-Elmagd, et al. (2004). "Graft versus host disease in intestinal transplantation." Am J Transplant 4(9): 14591465.

36. Moon, J. I., G. Selvaggi, et al. (2005). "Intestinal transplantation for the treatment of neoplastic disease." J Surg Oncol 92(4): 284-291.

37. Nishida, S., N. S. Hadjis, et al. (2004). "Intestinal and multivisceral transplantation after abdominal trauma." J Trauma 56(2): 323-327.

38. Okumura, M. and M. Mester (1992). "The coming of age of small bowel transplantation: a historical perspective." Transplant Proc 24(3): 1241-1242.

39. Pan, C., Y. Shi, et al. (2009). "Single-center experience of 253 portal vein thrombosis patients undergoing liver transplantation in China." Transplant Proc 41(9): 3761-3765.

40. Parizhskaya, M., C. Redondo, et al. (2003). "Chronic rejection of small bowel grafts: pediatric and adult study of risk factors and morphologic progression." Pediatr Dev Pathol 6(3): 240-250.

41. Petrisli, E., A. Chiereghin, et al. (2010). "Early and late virological monitoring of cytomegalovirus, Epstein-Barr virus, and human herpes virus 6 infections in small bowel/multivisceral transplant recipients." Transplant Proc 42(1): 74-78.

42. Selvaggi, G., D. Weppler, et al. (2007). "Ten-year experience in porto-caval hemitransposition for liver transplantation in the presence of portal vein thrombosis." Am J Transplant 7(2): 454460.

43. Starzl, T. E., M. I. Rowe, et al. (1989). "Transplantation of multiple abdominal viscera." JAMA 261(10): 1449-1457.

44. Sudan, D. (2006). "Cost and quality of life after intestinal transplantation." Gastroenterology 130(2 Suppl 1): S158-162.

45. Sudan, D. (2010). "Long-term outcomes and quality of life after intestine transplantation." Curr Opin Organ Transplant 15(3): 357360.

46. Tzakis, A. G., T. Kato, et al. (2005). "100 multivisceral transplants at a single center." Ann Surg 242(4): 480-490; discussion 491-483.

47. Vianna, R., R. O. Giovanardi, et al. (2005). "Multivisceral transplantation for diffuse portomesenteric thrombosis in a patient with life-threatening esophagogastroduodenal bleeding." Transplantation 80(4): 534-535.

48. Vianna, R. M., R. S. Mangus, et al. (2008). "Current status of small bowel and multivisceral transplantation." Adv Surg 42: 129-150.

49. Wu, T., K. Abu-Elmagd, et al. (2003). "A schema for histologic grading of small intestine allograft acute rejection." Transplantation 75(8): 1241-1248.

50. Yerdel, M. A., B. Gunson, et al. (2000). "Portal vein thrombosis in adults undergoing liver transplantation: risk factors, screening, management, and outcome." Transplantation 69(9): 1873-1881. 